\title{
Dagger categories and formal distributions
}

\author{
Richard Blute $^{1}$ and Prakash Panangaden ${ }^{2}$ \\ 1 Department of Mathematics \\ University of Ottawa \\ Ottawa, Ontario, Canada \\ rblute@uottawa.ca \\ 2 School of Computer Science \\ McGill University \\ Montreal, Quebec, Canada \\ prakash@cs.mcgill.ca
}

Summary. Monoidal dagger categories play a central role in the abstract quantum mechanics of Abramsky and Coecke. The authors show that a great deal of elementary quantum mechanics can be carried out in these categories; for example, the Born rule emerges naturally.

In this paper, we construct a category of tame formal distributions with coefficients in a commutative associative algebra and show that it is a dagger category. This gives access to a broad new class of models, with the abstract scalars in the sense of Abramsky being the elements of the algebra.

We will also consider a subcategory of local formal distributions, based on the ideas of Kac. Locality has been of fundamental significance in various formulations of quantum field theory. Thus our work may provide the possibility of extending the abstract framework to QFT.

We also show that these categories of formal distributions are monoidal and contain a nuclear ideal, a weak form of adjunction appropriate for analyzing categories such as the category of Hilbert spaces, where the nuclear maps are the HilbertSchmidt maps.

By taking formal distributions with coefficients in the dual of a cocommutative Hopf algebra, we obtain a categorical generalization of the Borcherds' notion of elementary vertex group.

\section{Introduction}

The Abramsky-Coecke notion of abstract quantum mechanics [2] is a proposal to abstract quantum theory away from the usual category of (possibly finitedimensional) Hilbert spaces and determine the underlying structures which should be taken as primitive. Unlike more traditional quantum logic, which is based on lattice theory, the Abramsky-Coecke approach is explicitly categorical in nature. The authors argue that the minimal necessary structure 
for interpreting quantum theory is that of a (monoidal) dagger category, i.e. a category with a strict involution which is the identity on objects.

They show that this framework provides a rich semantics for quantum computing and quantum information theory. For example, the Born rule emerges naturally from their axiomatization and one can express the correctness of various protocols, such as teleportation [7], as the commutativity of certain diagrams.

In subsequent work [3], the authors provide a diagrammatic language which simultaneously gives the free such category and provides a graphical language for reasoning about quantum systems.

Since their initial papers, the subject of abstract quantum theory and dagger categories has become quite active, and has developed important results. We mention in particular the work of Selinger [25]. Aside from developing another graphical language, the author considers the construction of completely positive maps in a general dagger category. CPMs are used, for example in the axiomatic description of quantum operations as described in [10]. (We also note that we use Selinger's notation and terminology throughout.)

Also important is the work of Coecke and Pavlovic [11], where they show that monoidal dagger categories even provide a framework for considering the existence of classical objects in a quantum universe. This is the subject of enormous research in quantum physics, see for example [15]. Furthermore, the description of classical structure in this setting is extremely elegant. A classical object in such a category is one with a compatible coalgebra structure. The comultiplication then models copying, and the counit models deleting, the two operations that define classical objects. Thus traditional algebraic/categorical structures are brought into consideration. See also [12] for further work in this direction.

Finally we mention Abramsky's paper [5]. Aside from summarizing much of the previous work discussed above, the author stresses the importance of abstract scalars. In any monoidal category, the scalars are the endomorphisms of the tensor unit. Traditionally, since quantum mechanics was carried out in the category of Hilbert spaces, the scalars were the complex numbers, this being the base field. But an abstract approach allows for considering other possibilities for scalars and Abramsky emphasizes the importance of being able to consider dagger categories with other choices for scalars. One of the interesting properties of the construction in this paper is that we will consider formal distributions with coefficients in an arbitrary commutative, associative algebra $A$, and the elements of $A$ will act as our scalars.

Monoidal dagger categories were considered by Abramsky, Blute and Panangaden [1] under the guise of tensored $*$-categories, (using teminology of Doplicher and Roberts [13]). We were interested in various extensions and elaborations of the category Rel of sets and binary relations. In particular, we were interested in developing a category whose objects are "continuously varying relations". So objects would be open subsets of Euclidean space, and morphisms would be continuous functions $\alpha: X \times Y \rightarrow \mathrm{C}$ (where $\mathrm{C}$ is the 
field of complex numbers.) Similarly, we wished to replace the usual relational composition:

$a(\mathcal{R} ; \mathcal{S}) c$ if and only if $\exists b$ such that $a \mathcal{R} b$ and $b \mathcal{S} c$

with the following "continuous analogue":

$$
(\alpha ; \beta)(x, z)=\int_{Y} \alpha(x, y) \beta(y, z) d y
$$

This idea led to the construction of the category DRel described below, and in [1]. Basically the objects of this category are open subsets of Euclidean space, and morphisms are certain well-behaved distributions. Distributions were introduced by Schwartz [24] to capture in a mathematically rigorous fashion the Dirac delta "function", which satisfied the relation

$$
(\alpha ; \delta)\left(x, y^{\prime}\right)=\int_{Y} \alpha(x, y) \delta\left(y, y^{\prime}\right) d y=\alpha\left(x, y^{\prime}\right)
$$

and its symmetric variant. In fact, no such function exists [6], though physicists made frequent use of such a $\delta$. As a recent example, quantum fields are today frequently modelled as operator-valued distributions in the Wightman axiomatization [18]. Schwartz axiomatizes the above $\delta$ as a generalized function or function with singularities. The distributions described in [1] are well-behaved in the sense that, when viewed as generalized functions, they have only mild singularities.

In this paper, we introduce a "formal" analogue of the DRel construction. Formal distributions, i.e. formal power series in both $x$ and $x^{-1}$, have played a fundamental role in algebraic and axiomatic approaches to quantum field theory. See, for example, [18, 22]. Indeed, they are the basis for the axiomatization of the notion of vertex algebra [18] and the notion of locality [18, 20], both of which figure in the present work. In this paper, we consider formal distributions with coefficients in a commutative algebra. We show that there is a formal notion of tameness inspired by the construction of DRel.

Previous work on formal distributions has focused on algebras of distributions. See for example the works $[18,19]$. However, in this paper, we wish to build a category of such distributions. In keeping with the passage from untyped to typed $\lambda$-calculus, we obtain a category by considering typed distributions. Atomic types are first assigned to the variables, and then a type for the distribution is inferred from these atomic types. One thus obtains a monoidal category, which we call ARel. We will see that the resulting category is a monoidal dagger category,

We also demonstrate that this category has a nuclear ideal, in the sense of [1]. In that paper, the authors observed that one of the key aspects of the category of sets and relations, the most elementary example of a monoidal dagger category, is that one has "transfer of variables" i.e. one can use the 
closed structure and the involution to move variables from "input" to "output". The category of Hilbert spaces does not allow such transfer of variables arbitrarily. Instead, one has a large class of morphisms which can be transposed in this fashion. These are the Hilbert-Schmidt maps. The notion of nuclear ideal captures the idea of "partially defined transpose". This idea was suggested by the definition of a nuclear morphism between Banach spaces, due to Grothendieck [16], and subsequent work of Higgs and Rowe [17]. Higgs and Rowe axiomatized the notion of nuclearity for a symmetric monoidal closed category, and is appropriate for the analysis of nuclearity for Banach spaces. The concept of nuclearity in analysis can be viewed as describing when one can think of linear maps as matrices. In the case of a compact closed dagger category such as Rel, all morphisms are nuclear, while in the category of Hilbert spaces, the nuclear morphisms are precisely the Hilbert-Schmidt maps [21].

In the category DRel discussed above, the Schwartz kernel theorem provides an inclusion of the space of test functions into the space of tame distributions, and such distributions form a nuclear ideal. Thus, another way of viewing the axioms of the definition of nuclear ideal is as an axiomitization of categories of (possibly) singular functions, containing a class of nonsingular functions. We show here that a formal analogue of this construction holds in our category ARel of formal distributions.

Another goal of this paper is to relate the notions arising in this paper and the vertex groups of Borcherds [9, 26]. Both can be viewed as axiomatizing the notion of singular map. In the former case, we have a category of singular maps, containing an ideal of nonsingular maps. In Borcherds' work, singular maps are viewed as an algebra over an algebra of nonsingular maps defined on some "group", (in fact, a Hopf algebra.). We show that when one considers the category of tame formal distributions with coefficients in the dual of a cocommutative Hopf algebra, one obtains examples of vertex categories, i.e. "many-object vertex groups".

The notion of (monoidal) dagger category has appeared in a number of guises. They appeared as tensored $*$-categories in the work of Doplicher and Roberts $[13,14]$. Their work involved considering categories of unitary representations of compact groups, one of the most significant examples of a monoidal dagger category. They considered such categories intheir analysis of superselection sectors, and proved a fundamental represnetation theorem. Any compact closed monoidal dagger category with certain normed structure (making it a $C^{*}$-category), is equivalent tothe category of representations of a compact group. Given the use of monoidal dagger categories and formal distributions in several axiomatizations of quantum field theory, it is our hope that the structures in this paper will be of use in extending the Abramsky-Coecke framework to include QFT. 


\section{Dagger categories and nuclear ideals}

We here review the crucial definitions of monoidal dagger category and nuclear ideal. See $[2,25,1]$ for more details, such as the appropriate coherence conditions.

Definition 2.1 A category $\mathcal{C}$ is a $\uparrow$-category if it is equipped with a functor $(-)^{\dagger}: \mathcal{C}^{o p} \rightarrow \mathcal{C}$, which is strictly involutive and the identity on objects. We will also assume our $\dagger$-categories are equipped with a conjugate functor $\overline{()}: \mathcal{C} \rightarrow \mathcal{C}$. A $\dagger$-category is $\dagger$-monoidal if it is symmetric monoidal, $(f \otimes g)^{\dagger}=f^{\dagger} \otimes g^{\dagger}$, and the conjugate functor has natural isomorphisms $\overline{\bar{A}} \cong A, \overline{A \otimes B} \cong \bar{A} \otimes \bar{B}$, and $\bar{I} \cong I$. (We will generally take these to be equalities.) These must satisfy evident equations, see [25].

Definition 2.2 Let $\mathcal{C}$ be a monoidal $\dagger$-category. A nuclear ideal for $\mathcal{C}$ consists of the following structure:

- For all objects $A, B \in \mathcal{C}$, a subset $\mathcal{N}(A, B) \subseteq \operatorname{Hom}(A, B)$. We will refer to the union of these subsets as $\mathcal{N}(\mathcal{C})$ or $\mathcal{N}$. We will refer to the elements of $\mathcal{N}$ as nuclear maps. The class $\mathcal{N}$ must be closed under composition with arbitrary $\mathcal{C}$-morphisms, closed under $\otimes$, closed under ()$^{\dagger}$, and the conjugate functor.

- A bijection $\theta: \mathcal{N}(A, B) \rightarrow \operatorname{Hom}(I, \bar{A} \otimes B)$. The bijection $\theta$ must be natural and preserve the $\dagger$-monoidal structure in an evident sense, see [1].

\section{Examples}

- The category Rel of sets and relations is a monoidal dagger category for which the entire category forms a nuclear ideal. Indeed any compact monoidal dagger category has this property.

- The category Hilb of Hilbert spaces and bounded linear maps maps is a well-known monoidal dagger category, which, in fact, led to the axiomatization [13]. Then the Hilbert-Schmidt maps form a nuclear ideal [1]. (This is one of the only examples where the conjugate functor is not merely the identity. Here it is the conjugate Hilbert space.)

- The category DRel of tame distributions on Euclidean space is a monoidal dagger category. The ideal of test functions (viewed as distributions) is a nuclear ideal. See [1] or the next section.

- We will define a subcategory of Rel called the category of locally finite relations. Let $R: A \rightarrow B$ be a binary relation and $a \in A$. Then let $R_{a}=$ $\{b \in B \mid a R b\}$. Define $R_{b}$ similarly for $b \in B$. Then we say that a relation is locally finite if, for all $a \in A, b \in B, R_{a}, R_{b}$ are finite sets. Then it is straightforward to verify that we have a monoidal dagger category which is no longer compact closed. It is also easy to verify that the finite relations form a nuclear ideal. 


\section{Distributions as Relations}

In this section, we review the construction of the category of tame distributions, denoted DRel [1]. We assume familiarity with basic notions from distribution theory. Suitable references are $[24,27,6]$.

The idea was to build a category where composition is given by the formula:

$$
\varphi(x, y) ; \psi(y, z)=\int \varphi(x, y) \psi(y, z) d y .
$$

The intuition that guided our original work was that integration should generalize the existential quantification that appears in the definition of relational composition. The proper framework for constructing such a category is the theory of distributions. Recall that if $\Omega$ denotes a nonempty open subset of $\mathrm{R}^{n}$, then $\mathcal{D}(\Omega)$ denotes the smooth (complex-valued) functions of compact support on $\Omega$. We will refer to the elements of $\mathcal{D}(\Omega)$ as test functions. $\mathcal{D}(\Omega)$ is given the structure of a topological vector space [6,27]. Then a distribution on $\Omega$ is a continuous, linear (complex-valued) functional on $\mathcal{D}(\Omega)$. Let $\mathcal{D}^{\prime}(\Omega)$ denote the space of all distributions on $\Omega$, equipped with the topology of pointwise convergence. We have a canonical inclusion

$$
\iota: \mathcal{D}(X) \hookrightarrow \mathcal{D}^{\prime}(X)
$$

given as follows:

$$
\phi(x) \mapsto\left[\psi(x) \in \mathcal{D}(X) \mapsto \int \phi(x) \psi(x) d x\right]
$$

This inclusion prompts one to consider distributions as singular or generalized functions. The $\delta$-function is often explicitly described this way. Also adding to the strength of this interpretation are results showing that arbitrary distributions can be obtained as limits of test functions. The representation of the $\delta$-function in this way is of special interest. See [6].

There is a canonical (dense) inclusion of $\mathcal{D}(X) \otimes \mathcal{D}(Y)$ into $\mathcal{D}(X \times Y)$ given by:

$$
\varphi \otimes \psi \mapsto[(x, y) \mapsto \varphi(x) \psi(y)]
$$

The construction of DRel also makes essential use of the Schwartz kernel theorem. We need the following notations to state the theorem. If $f$ is a distribution on $X \times Y$ and $\phi \in \mathcal{D}(X)$ then $f_{*}(\phi)$ will be the function from $\mathcal{D}(Y)$ to the base field given by $\psi \in \mathcal{D}(Y) \mapsto f(\phi \otimes \psi)$ and $f^{*}(\psi)$ is the map $\phi \in \mathcal{D}(X) \mapsto f(\phi \otimes \psi)$ The Schwartz kernel theorem states [27]:

Theorem 3.1 Let $X$ and $Y$ be two open subsets of $\mathrm{R}^{n}$ and $\mathrm{R}^{m}$. Let $f$ be a distribution on $X \times Y$. For all functions $\phi \in \mathcal{D}(X)$ the linear map $f_{*}(\phi)$ is a distribution on $Y$. Furthermore, the map $\phi \mapsto f_{*}(\phi)$ from $\mathcal{D}(X)$ to $\mathcal{D}^{\prime}(Y)$ is continuous. There is also a converse to this statement. 
Definition 3.2 A tame distribution on $X \times Y$ is a distribution $f$ on $X \times Y$ such that each of $f^{*}$ and $f_{*}$ factor continuously through the appropriate $\iota$, where $\iota$ is the inclusion of the space of test functions into the space of distributions. Explicitly, there exist continuous linear maps

$$
f_{L}: \mathcal{D}(X) \rightarrow \mathcal{D}(Y) \quad f_{R}: \mathcal{D}(Y) \rightarrow \mathcal{D}(X)
$$

such that for every $\phi \in \mathcal{D}(X)$ and $\psi \in \mathcal{D}(Y)$, we have:

$$
f_{*}(\phi)(\psi)=f^{*}(\psi)(\phi)=f(\phi \otimes \psi)=\int f_{L}(\phi) \psi d y=\int \phi f_{R}(\psi) d x
$$

We write $\mathcal{T}(X, Y)$ for the tame distributions on $X \times Y$.

Intuitively, tame distributions are allowed to be mildly singular, in that composing with a test function "tames" the singularity.

\section{Examples}

- Let $X$ be an open subset of $\mathrm{R}^{n}$. The trace distribution on $X \times X$ is given by $\operatorname{Tr}(\eta)=\int \eta(x, x) d x$ where $\eta\left(x, x^{\prime}\right) \in \mathcal{D}(X \times X)$. From this definition it follows that $\operatorname{Tr}_{*}(\phi)(\psi)=\operatorname{Tr}^{*}(\psi)(\phi)=\operatorname{Tr}(\phi \otimes \psi)=\int \phi(x) \psi(x) d x$. Thus we clearly have $\operatorname{Tr}_{L}(\phi)=\operatorname{Tr}_{R}(\phi)=\phi$, which shows that $\delta$ is tame. This tame distribution will act as the identity in our category.

- A regular distribution on $X$ is a distribution of the form

$$
\alpha(x) \mapsto \int_{X} \alpha(x) \beta(x)
$$

for some fixed function $\beta$ on $X$. $\beta$ is called the kernel of the distribution. Suppose that $T$ is a regular distribution on $X \times Y$ with a test function $\beta(x, y)$ as its kernel, that is to say:

$$
T(\alpha(x, y))=\int_{X \times Y} \beta(x, y) \alpha(x, y)
$$

Then $T$ is tame with its associated functions being given by:

$$
T_{L}(\phi)=\int_{X} \beta(x, y) \phi(x) \quad T_{R}(\psi)=\int_{Y} \beta(x, y) \psi(y)
$$

We denote the space of all such distributions by $\mathcal{N}(Y, Z)$.

Given tame distributions we can define the following operation which will serve as composition. Suppose that $f \in \mathcal{T}(X, Y), g \in \mathcal{T}(Y, Z)$. We define $f ; g \in \mathcal{T}(X, Z)$ as follows. Given that $f$ is tame, we have a continuous function $f_{L}: \mathcal{D}(X) \rightarrow \mathcal{D}(Y)$. Applying the Schwartz kernel theorem to $g$, we obtain a morphism $g_{*}: \mathcal{D}(Y) \rightarrow \mathcal{D}^{\prime}(Z)$. Composition gives a continuous map $\mathcal{D}(X) \rightarrow$ $\mathcal{D}^{\prime}(Z)$. By the kernel theorem again, we obtain a distribution on $X \times Z$. 
Definition 3.3 The category DRel has as objects open subsets on $\mathrm{R}^{n}$, and, as morphisms, tame distributions. Composition is as described above.

Theorem 3.4 DRel is a monoidal †-category.

The tensor product is given as follows. Given objects $X$ and $Y$ we define $X \otimes Y$ as the cartesian product space $X \times Y$. Given morphisms in DRel $f: X \rightarrow Y$ and $g: X^{\prime} \rightarrow Y^{\prime}$ we can define $f \otimes g: X \otimes X^{\prime} \rightarrow Y \otimes Y^{\prime}$ as follows. We first define $f \otimes g$ as a distribution on $\mathcal{D}(X) \otimes \mathcal{D}\left(X^{\prime}\right) \otimes \mathcal{D}(Y) \otimes \mathcal{D}\left(Y^{\prime}\right)$ by the formula $(f \otimes g)\left(\phi(x) \otimes \phi^{\prime}\left(x^{\prime}\right) \otimes \psi(y) \otimes \psi^{\prime}\left(y^{\prime}\right)\right)=f(\phi \otimes \psi) g\left(\phi^{\prime} \otimes \psi^{\prime}\right)$. It is routine to verify that this is tame. Finally the $\dagger$-structure is the identity on objects. On morphisms, the only thing that changes is the role of $f_{L}$ and $f_{R}$. The conjugate functor is taken to be the identity. We then have:

Theorem 3.5 The sets $\mathcal{N}(Y, Z)$ form a nuclear ideal for DRel.

\section{Categories of formal distributions}

We now review the basic theory of formal distributions. Much of this theory was developped by Kac. Suitable references are $[18,19]$. In the following, $A$ will always denote a commutative, associative, unital algebra over some field $k$.

An expression of the form $\alpha(z)=\Sigma_{n \in Z} \alpha_{n} z^{n}$, where $Z$ is the set of integers, $\alpha_{n} \in A$ and $z$ is a variable, is called a formal distribution with coefficients in $A$. Similarly, one can speak of formal distributions in several variables. The set of formal distributions in a fixed set of variables forms an infinite dimensional vector space, denoted $A\left[\left[z, z^{-1}, w, w^{-1}, \ldots\right]\right]$.

The space of distributions has a great deal of structure, much of which is analogous to Schwartz's original theory of distributions. The key to defining such structure is the residue operation, defined by $\operatorname{Res}_{z}(\alpha(z))=\alpha_{-1} \in$ $A$, i.e. the residue of $\alpha$ is the coefficient of $z^{-1}$. Similarly, if $\alpha(z, w) \in$ $A\left[\left[z, z^{-1}, w, w^{-1}\right]\right]$, we can define $\operatorname{Res}_{z}(\alpha(z, w)) \in A\left[\left[w, w^{-1}\right]\right]$.

We now observe that the space of Laurent polynomials $A\left[z, z^{-1}\right]$ can be viewed as the test functions for these formal distributions, with the evaluation $A\left[\left[z, z^{-1}\right]\right] \times A\left[z, z^{-1}\right] \rightarrow A$ being defined by

$$
<\alpha(z), f(z)>=\operatorname{Res}_{z} f(z) \alpha(z)
$$

There is a formal analogue of the injection $\mathcal{D}(Y) \rightarrow \mathcal{D}^{\prime}(Y)$ which is given simply by the inclusion $A\left[y, y^{-1}\right] \subseteq A\left[\left[y, y^{-1}\right]\right]$, and similarly in the multivariable case. There is a corresponding version of the Shwartz kernel theorem as well.

The formal Dirac delta is given by the distribution:

$$
\delta(z, w)=z^{-1} \sum_{n \in Z}\left(\frac{z}{w}\right)^{n}
$$


We have the fundamental property that for all $f(z) \in A\left[z, z^{-1}\right]$

$$
<\delta(z, w), f(z)>=f(w)
$$

Note that, in this equation, we are multiplying two distributions. In general, this cannot be done even formally, due to the possibility of infinite coefficients. We must have a notion of "tameness" to perform such multiplications. We will see that the Dirac delta is indeed tame.

One can also reiterate the process of taking residues. If $\alpha$ is a distribution, and $x_{1}, x_{2}, \ldots, x_{n}$ are among its variables, then we define

$$
\left.\operatorname{Res}_{x_{1}, x_{2}, \ldots, x_{n}} \alpha=\operatorname{Res}_{x_{1}}\left(\operatorname{Res}_{x_{2}}\left(\ldots \operatorname{Res}_{x_{n}} \alpha\right)\right) \ldots\right)
$$

One can readily check that this is well-defined and independent of the order in which the residues are taken.

We also note that the space of formal distributions allows formal differentiation, i.e. we have operators:

$$
\partial=\partial_{z}: A\left[\left[z, z^{-1}, w, w^{-1}, \ldots\right]\right] \rightarrow A\left[\left[z, z^{-1}, w, w^{-1}, \ldots\right]\right]
$$

and that these satisfy equations analogous to those for differentiation of distributions, e.g.

$$
\operatorname{Res}_{z} \partial \alpha(z) \beta(z)=-\operatorname{Res}_{z} \alpha(z) \partial \beta(z)
$$

This is a formal analogue of integration by parts. Consult [18] for these and other results, such as the representation of distributions in terms of derivatives of deltas.

\subsection{Tameness for formal distributions}

We will now define a category which will be the formal analogue of DRel, and this category will have much of the same structure. We assume throughout the remainder of this section that $\mathcal{A}$ is a fixed associative unital algebra over a field $k$.

We assume the existence of an infinite set of atomic types. These will be type variables denoted $A_{1}, A_{2}, B, \ldots$. Then the set of all types consists of all words of the form $A_{1} \otimes A_{2} \ldots \otimes A_{n}$. We refer to $n$ as the length of the word. We also assume the existence of a unique word of length 0 , denoted $I . I$ is the tensor unit, and acts as the identity in the monoid of words. (Thus we will be working in a strict monoidal category). We also assume that we have an infinite stock of variables for each atomic type. These will be denoted $x: A$, but we will generally not write the type, if there is no danger of confusion.

Now we can talk about typed distributions. A formal distribution of type $A_{1} \otimes A_{2} \ldots \otimes A_{m}$ is an element of $\mathcal{A}\left[\left[x_{1}, x_{1}^{-1}, \ldots, x_{m}, x_{m}^{-1}\right]\right]$, where $x_{i}$ is of type $A_{i}$. We say that a formal distribution $\alpha\left(x_{1}, x_{2}, \ldots, x_{m}, y_{1}, y_{2}, \ldots, y_{n}\right)$ of 
type $A_{1} \otimes A_{2} \ldots A_{m} \otimes B_{1} \otimes \ldots B_{n}$ is tame with respect to the type splitting $A_{1} \otimes A_{2} \ldots A_{m} \| B_{1} \otimes \ldots B_{n}$ if, for all $f \in \mathcal{A}\left[x_{1}, x_{1}^{-1}, \ldots, x_{m}, x_{m}^{-1}\right]$,

$$
\operatorname{Res}_{x_{1}, x_{2}, \ldots, x_{m}}(f \alpha) \in \mathcal{A}\left[y_{1}, y_{1}^{-1}, \ldots, y_{n}, y_{n}^{-1}\right]
$$

and dually for all $g \in \mathcal{A}\left[y_{1}, y_{1}^{-1}, \ldots, y_{n}, y_{n}^{-1}\right]$.

In other words, a tame distribution takes Laurent polynomials to Laurent polynomials. This is the obvious analogue of the notion of tameness used in [1], given that in the formal setting we are using Laurent polynomials as test functions.

Remark 4.1 We note that we consider two distributions (of the same type) equivalent if they are identical up to $\alpha$-conversion, i.e. up to change of variable name (within the same type).

We are now ready to define the category ARel.

Definition 4.2 The category ARel is defined as follows. Objects are types. A morphism

$\alpha: A_{1} \otimes A_{2} \otimes \ldots \otimes A_{n} \rightarrow B_{1} \otimes B_{2} \ldots B_{m}$ is (the equivalence class of) a distribution of type $A_{1} \otimes A_{2} \otimes \ldots \otimes A_{n} \otimes B_{1} \otimes B_{2} \ldots B_{m}$ which is tame with respect to the type splitting $A_{1} \otimes A_{2} \otimes \ldots \otimes A_{n} \| B_{1} \otimes B_{2} \ldots B_{m}$ Composition is defined as follows. Suppose that $\alpha: A_{1} \otimes A_{2} \otimes \ldots \otimes A_{n} \rightarrow B_{1} \otimes B_{2} \ldots B_{m}$ and that $\beta: B_{1} \otimes B_{2} \otimes \ldots \otimes B_{m} \rightarrow C_{1} \otimes C_{2} \ldots \otimes C_{p}$. Then we have

$$
\begin{gathered}
\beta \alpha\left(x_{1}, x_{2}, \ldots, x_{n}, y_{1}, \ldots, y_{p}\right)= \\
\operatorname{Res}_{z_{1}, z_{2}, \ldots, z_{m}}\left[\alpha\left(x_{1}, \ldots, x_{n}, z_{1}, \ldots, z_{m}\right) \beta\left(z_{1}, \ldots, z_{m}, y_{1}, \ldots, y_{p}\right)\right]
\end{gathered}
$$

Note that one must always be careful to use distinct variables in the two distributions being composed.

The identity is defined as:

$$
i d: A_{1} \otimes A_{2} \otimes \ldots \otimes A_{n} \rightarrow A_{1} \otimes A_{2} \otimes \ldots \otimes A_{n}=\Pi_{i=1}^{n} \delta_{A_{i}}
$$

Also note that we set $\operatorname{Hom}(I, I)=\mathcal{A}$, and more generally $\operatorname{Hom}(I, A)$ is the space of Laurent polynomials on $A$. The justification for this is as in [1].

Theorem 4.3 ARel is a category.

Proof. There are a number of things to check here, most are more or less straightforward. One must check that $\delta$ is tame, and that the product of $\delta$ 's does indeed act as identity. One must check that the composite of two tame distributions is again tame, and finally associativity of composition follows from the observation that $\alpha \operatorname{Res}_{z} \beta=\operatorname{Res}_{z} \alpha \beta$, when $z$ is not among $\alpha$ 's variables. 
Theorem 4.4 ARel is a monoidal $\dagger$-category.

Proof. The tensor on objects is obvious. On morphisms, the tensor is given by multiplication. Again, when multiplying two distributions together, one must always make sure that the two distributions use distinct variables. The conjugate functor is taken to be the identity, and the $\dagger$-functor reverses the order of variables. The necessary equations are straightforward to verify.

Finally, we may state the following result which is also straightforward.

Theorem 4.5 The Laurent polynomials form a nuclear ideal for ARel.

Proof. The bijection $\theta: \mathcal{N}(A, B) \rightarrow \operatorname{Hom}(I, \bar{A} \otimes B)$ is the obvious injection of the test functions into the corresponding space of distributions. The necessary equations are all evident.

\subsection{Locality for formal distributions.}

We now review one of the crucial topics in formal distribution theory, the notion of locality of a formal distribution. This notion has been emphasized heavily by $\operatorname{Kac}[18,19,20]$. These are a fundamental class of distributions which were inspired by the notion of locality in quantum field theory.

Definition 4.6 A formal distribution $\alpha(x, y)$ is local if there exists a positive integer $N$ such that $(x-y)^{N} \alpha(x, y)=0$.

The formal Dirac delta is local, as $(x-y) \delta(x, y)=0$. Similarly, any derivative of the delta is local. We here collect some basic identities on derivatives of delta which are useful in proving such results.

Lemma 4.7 ([18], p. 16)

- $\delta(x, y)=\delta(y, x)$

- $\partial_{x}^{j} \delta(x, y)=\left(-\partial_{y}\right)^{j} \delta(x, y)$

- $(x-y)^{j+1} \partial_{x}^{j} \delta(x, y)=0$

Now with the above formulas, one may characterize completely the local formal distributions:

Theorem 4.8 (Kac [18],p.18) The local distributions are precisely those of the form:

$$
\alpha(x, y)=\sum_{j \in Z^{+}} c^{j}(y) \partial_{y}^{(j)} \delta(x, y)
$$

where the above sum is finite and $c^{j}(y)=\mathcal{A}\left[\left[y, y^{-1}\right]\right]$. The series $c^{j}(y)$ can be calculated by the formula:

$$
c^{j}(y)=\operatorname{Res}_{x} \alpha(x, y)(x-y)^{j}
$$


It is now straightforward to verify that the tame local distributions form a $\dagger$-subcategory. The only thing remaining to verify is the following:

Lemma 4.9 Suppose that $\alpha(x, y)$ and $\beta(y, z)$ are tame local distributions. Then $\operatorname{Res}_{y}[\alpha(x, y) \beta(y, z)]$ is local as well. (In particular, it is well-defined.)

Proof. This follows from the above characterization of local distributions, and repeated application of the "integration by parts" formula.

Now we define a category Loc-ARel, whose objects are atomic formal types, and morphisms are local distributions. Loc-ARel has an evident $\dagger$-category structure.

\subsection{Monoidal structure for Loc-ARel}

We now describe a tensor structure for the category Loc-ARel. This first requires defining an $n$-ary version of locality:

Definition 4.10 We suppose that $\alpha: A_{1} \otimes A_{2} \otimes \ldots A_{n} \rightarrow B_{1} \otimes B_{2} \otimes$ $\ldots B_{n}$ is a tame distribution, and that the corresponding variables are $x_{1}, x_{2}, \ldots, x_{n}, y_{1}, y_{2}, \ldots, y_{n}$. Then we say that $\alpha$ is local if there is a permutation $\sigma$ of the set $\{1,2, \ldots, n\}$ such that for all $i \in\{1,2, \ldots, n\}$, there exists a natural number $N_{i}$ such that:

$$
\left(x_{i}-y_{\sigma(i)}\right)^{N_{i}} \alpha=0
$$

Lemma 4.11 Loc-ARel is a monoidal †-subcategory of ARel.

Note however that there is no longer a nuclear ideal. However a slight modification of the notion of locality does yield a subcategory with a nuclear ideal. We say that a tame distribution $\alpha: A \rightarrow B$ is stable if it is of the form $\alpha=\alpha_{1}+\alpha_{2}$, where $\alpha_{1}$ is (tame) local, and $\alpha_{2}$ is a Laurent polynomial. Thus the stable distributions only fail slightly to be local. It is straightforward to verify that we indeed have a category.

Lemma 4.12 Let $\alpha(x, y)$ and $\beta(y, z)$ be stable distributions. Then $\operatorname{Res}_{y}[\alpha(x, y) \beta(y, z)]$ is as well.

Proof. One simply notes that the composition of two tame local distributions is tame and local, the composition of two nuclear morphisms is nuclear, and the compostion of a tame local distribution and a nuclear distribution is nuclear. The result now follows from the bilinearity of composition.

So we define a category S-ARel whose objects are formal types and morphisms are stable distributions. It is evidently a monoidal †-subcategory of ARel. S-ARel is essentially the smallest extension of Loc-ARel for which there is a nuclear ideal.

Theorem 4.13 The Laurent polynomials form a nuclear ideal in S-ARel. 


\section{Vertex groups and categories}

In this section, we review Borcherds' notion of an elementary vertex group [9], and then give a minor generalization of this notion, that being the notion of a vertex category, i.e. a many-object vertex group. We demonstrate that the category ARel of the previous section gives an example of a vertex category, whenever $\mathcal{A}$ is taken to be the dual of a cocommutative Hopf algebra $H$. We show further that when considering the algebra determined by the endomorphisms of an object of a vertex category, one obtains a vertex group in the Borcherds sense. We first review some basic facts about duals of Hopf algebras.

Before getting into the technical details of vertex groups, we recall some facts about duals of Hopf algebras. See [23] for details.

First recall that if $H$ is a Hopf algebra, then $H^{*}$, the linear dual of $H$, is generally not a Hopf algebra, unless $H$ is finite-dimensional. However, we have:

Lemma 5.1 The dual of the comultiplication $\Delta: H \rightarrow H \otimes H$ induces an algebra structure on $H^{*}$, when composed with the canonical inclusion $H^{*} \otimes$ $H^{*} \rightarrow(H \otimes H)^{*}$. If $H$ is cocommutative, then $H^{*}$ is a commutative algebra. Thus, if $f, g \in H^{*}$ and $h \in H$, then

$$
(f g)(h)=\sum_{h} f\left(h_{1}\right) g\left(h_{2}\right)
$$

using the usual Sweedler notation, i.e.

$$
\Delta(h)=\sum_{h} h_{1} \otimes h_{2}
$$

We will also make use of the fact that $H^{*}$ has a canonical structure as a two-sided $H$-module via the formulas:

$$
(h f)\left(h^{\prime}\right)=f\left(h^{\prime} h\right) \quad(f h)\left(h^{\prime}\right)=f\left(h h^{\prime}\right)
$$

Remark 5.2 Finally we note that the existence of an involutive antipode gives a second possible monoidal $\dagger$-structure on ARel. If $\alpha(x, y)=\sum \alpha_{i j} x^{i} y^{j}$ is a morphism from $A$ to $B$, then define

$$
\bar{\alpha}=\sum S^{*}\left(\alpha_{i j}\right) x^{i} y^{j}
$$

and

$$
\alpha^{\dagger}=\sum S^{*}\left(\alpha_{i j}\right) y^{j} x^{i}
$$

In this section, we will always mean this monoidal $\dagger$-structure. 
The following definition is due to Borcherds [9]. It has been studied and elaborated on extensively by Snydal [26]. For examples, see either of these two references.

Definition 5.3 Let $H$ be a cocommutative Hopf algebra over a field $k$. A vertex group on $H$ consists of a $k$-vector space $K$, the ring of singular functions on $H$, with the following additional structure:

- $K$ is an associative, unital algebra over the algebra $H^{*}$.

- $K$ is a two-sided $H$-module. Further, the unit map $\eta: H^{*} \rightarrow K$ is a map of 2 -sided $H$-modules.

- The product map on $K, \mu: K \otimes K \rightarrow K$ is equivariant under the left and right actions of $H$.

- There is a morphism $S_{K}: K \rightarrow K$ such that $S_{K} \circ \eta=\eta \circ S^{*}$.

We further require that $S_{K}$ be an antialgebra map, and that $S_{K}^{2}=i d$. If the algebra $K$ is also commutative, then we say that we have a commutative vertex group.

Borcherds and Snydal only consider the commutative case, but the present work yields several natural noncommutative examples.

We now provide a categorical generalization of the previous definition by introducing the notion of a vertex category. This is the correct generalization in that a one-object vertex category is indeed a vertex group.

Definition 5.4 Let $H$ be a cocommutative Hopf algebra. An $H$-vertex category consists of a $\dagger$-category $\mathcal{C}$ such that:

- For all objects $A, B$ in $\mathcal{C}$, we have that $\operatorname{Hom}(A, B)$ is an $H^{*}$ - module, a 2-sided $H$-module, and composition is $H^{*}$-bilinear.

- Composition also satisfies the following $H$-invariance property: If $f: A \rightarrow$ $B$ and $g: B \rightarrow C$, then we have ( $\triangleright$ and $\triangleleft$ denote the actions of $H$.)

$$
\begin{aligned}
& h \triangleright(g f)=\Sigma_{h}\left(h_{1} \triangleright g\right)\left(h_{2} \triangleright f\right) \\
& (g f) \triangleleft h=\Sigma_{h}\left(g \triangleleft h_{1}\right)\left(f \triangleleft h_{2}\right)
\end{aligned}
$$

- We must also have the following antipode condition. First note that there is a canonical morphism $\eta: H^{*} \rightarrow \operatorname{Hom}(A, A)$ which takes $f \in H^{*}$ to $f \triangleright i d$. We require that $\eta$ be a map of $H$-modules and that the following diagram commutes.

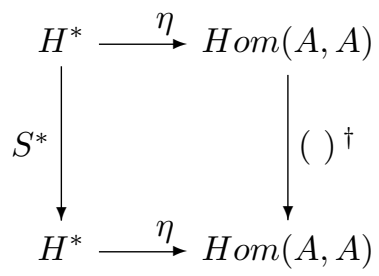


The following results are all straightforward. All actions are defined by acting on coefficients.

Theorem 5.5 - A one-object vertex category is a vertex group, with $S_{K}$ being given by the dagger operation on Homsets.

- When $A$ is the dual of a cocommutative Hopf algebra, then ARel is a vertex category.

- In any vertex category $\mathrm{C}$, if $C \in \mathrm{C}$, then $\operatorname{Hom}(C, C)$ is a vertex group.

\section{Conclusion}

The primary goal of the theory of formal distributions is to develop a more purely algebraic version of the Schwartz theory of distributions. Then the issue becomes the extent to which the original theory lifts to the algebraic setting. This is for example one of the goals of the monograph [18]. One is particularly interested in the many applications of distribution theory in quantum physics. In this paper, we have shown that the structure of the category DRel lifts to this formal setting in a straightforward way. Thus one is able to view these formal distributions as generalized relations, as discussed in [1]. We hope to explore this idea in the future.

Along the same lines, we have introduced the notion of a vertex category or multiobject vertex algebra. Connecting this idea with the original work of Borcherds [9] and Snydal [26] is also work we intend to explore.

\section{References}

1. S. Abramsky, R. Blute, P. Panangaden. Nuclear and trace ideals in tensored *-categories. Journal of Pure and Applied Algebra, 143:3-47, 1999.

2. S. Abramsky, B. Coecke. A categorical semantics of quantum protocols, in Proceedings of the 19th Annual IEEE Symposium on Logic in Computer Science: LICS 2004, IEEE Computer Society, 415-425, 2004.

3. S. Abramsky, B. Coecke. Abstract physical traces, Theory and Applications of Categories 14:111-124, 2005.

4. S. Abramsky, R. Jagadeesan. New foundations for the geometry of interaction. Information and Computation, 111(1):53-119,1994.

5. S. Abramsky. Abstract scalars, loops, and free traced and strongly compact closed categories, in Proceedings of CALCO 2005, Springer Lecture Notes in Computer Science Vol. 3629, 1-31, 2005.

6. M. A. Al-Gwaiz. Theory of Distributions. Dekker Pure and Applied Mathematics, 1992.

7. C. Bennett, G. Brassard, C. Crpeau, R. Jozsa, A. Peres, and W. Wootters. Teleporting an unknown quantum state via dual classical and Einstein-PodolskyRosen channels, Physical Review Letters 70: 1895 - 1899, (1993).

8. R.F. Blute, J.R.B. Cockett, R.A.G. Seely, Feedback for linearly distributive categories: traces and fixpoints, Journal of Pure and Applied Algebra, 154:27$69,(2000)$ 
9. R. Borcherds. Vertex algebras. preprint, q-alg/9706008. Also appeared in Topological field theory, primitive forms and related topics (Kyoto, 1996) Progress in Mathematics Volume 60, Birkhäuser Boston, (1998).

10. I. Chuang, M. Nielsen, Quantum Computation and Quantum Information, Cambridge University Press, 2000.

11. B. Coecke, D. Pavlovic. Quantum measurements without sums, to appear in The Mathematics of Quantum Computation and Technology; Chen, Kauffman and Lomonaco (eds.), 2006.

12. B. Coecke, E. Paquette, D. Pavlovic, POVMs and Naimark's theorem without sums, preprint, 2006.

13. S. Doplicher and J. Roberts. A new duality theory for compact groups. Inventiones Mathematicae, 98:157-218, 1989.

14. P. Ghez, R. Lima, and J. Roberts. $w^{*}$-categories. Pacific Journal of Mathematics, 120:79-109, 1985.

15. D. Giulini, E. Joos, C. Kiefer, J. Kupsch, I.-O. Stamatescu, H.D. Zeh. Decoherence and the Appearance of a Classical World in Quantum Theory, SpringerVerlag, 1996.

16. A. Grothendieck. Produits Tensoriels Topologiques et Espaces Nucleaires. AMS Memoirs. American Mathematical Society, 1955.

17. D. A. Higgs, K. Rowe. Nuclearity in the category of complete semilattices. Journal of Pure and Applied Algebra, 57:67-78, 1989.

18. V. Kac. Vertex algebras for beginners. University Lecture Series, American Mathematical Society, 1997.

19. V. Kac. Formal distributions algebras and conformal algebras, XIIth International Congress of Mathematical Physics, 1997, p.80-97, q-alg/9709027.

20. V. Kac. The idea of locality, preprint q-alg/9709008.

21. R. Kadison, J. Ringrose. Fundamentals of the Theory of Operator Algebras. Academic Press, 1983.

22. B. Lian, G. Zuckerman. Commutative quantum operator algebras. Journal of Pure and Applied Algebra, 100:117-141 (1995)

23. S. Majid. Foundations of Quantum Group Theory. Cambridge University Press, 1995.

24. L. Schwartz. Théorie des Distributions. Hermann, 1957.

25. P. Selinger. Dagger compact closed categories and completely positive maps. in Proceedings of the 3rd International Workshop on Quantum Programming Languages (QPL 2005), Chicago. ENTCS 170:139-163, 2007.

26. C. Snydal. Equivalence of Borcherds $G$-vertex algebras and axiomatic vertex algebras, preprint math.QA/9904104.

27. F. Treves. Topological Vector Spaces, Distributions and Kernels. Pure and Applied Mathematics 25. Academic Press, 1967. 